\title{
A thermodynamically stable nanophase material
}

\section{-Supplementary Information}

Zhang Lin $^{1}$, Benjamin Gilbert ${ }^{2}$, Quanlin Liu $^{3}$, Guoqiang Ren ${ }^{1}$, Feng Huang ${ }^{1, *}$

1. Fujian Institute of Research on the Structure of Matter, National Engineering Research Center for Optoelectronic Materials, Chinese Academy of Sciences, Fuzhou, Fujian, 350002, People's Republic of China

2. Earth Sciences Division, Lawrence Berkeley National Laboratory, Berkeley, CA 94720, U.S. A

3. Department of Materials Physics and Chemistry, University of Science and Technology Beijing, Beijing 100083, People's Republic of China

*Corresponding author: fhuang@fjirsm.ac.cn 

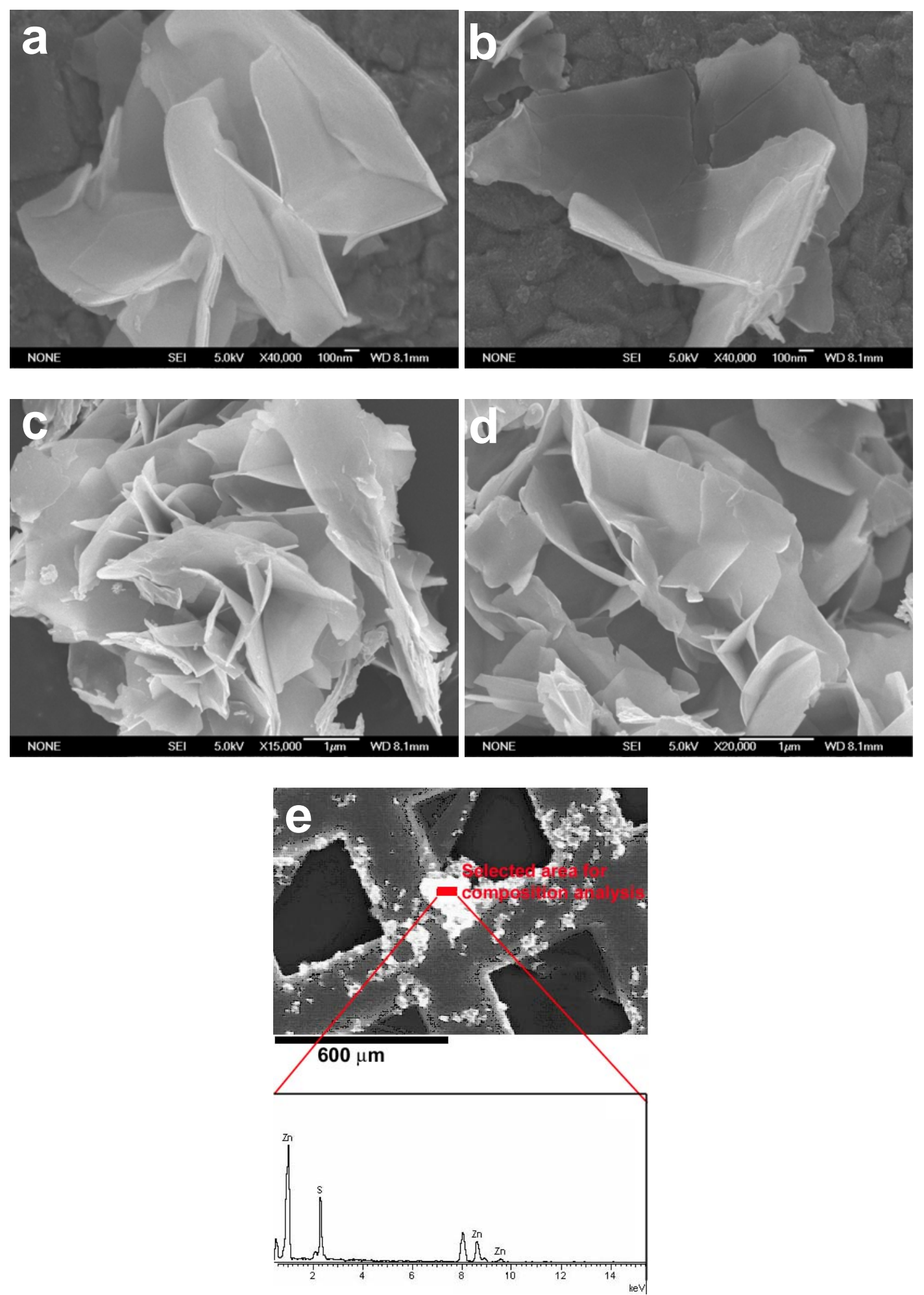

Supplementary Figure S1

Supplementary Figure S1 a),b),c),d) Low-magnification images from SEM showing the homogeneity of the sample in nanosheet morphology. e) Selected area element analysis of the nanosheet aggregates showing only Zn, and S elements are present in the sample. 


\section{Indexing the nanosheet polytype structure}
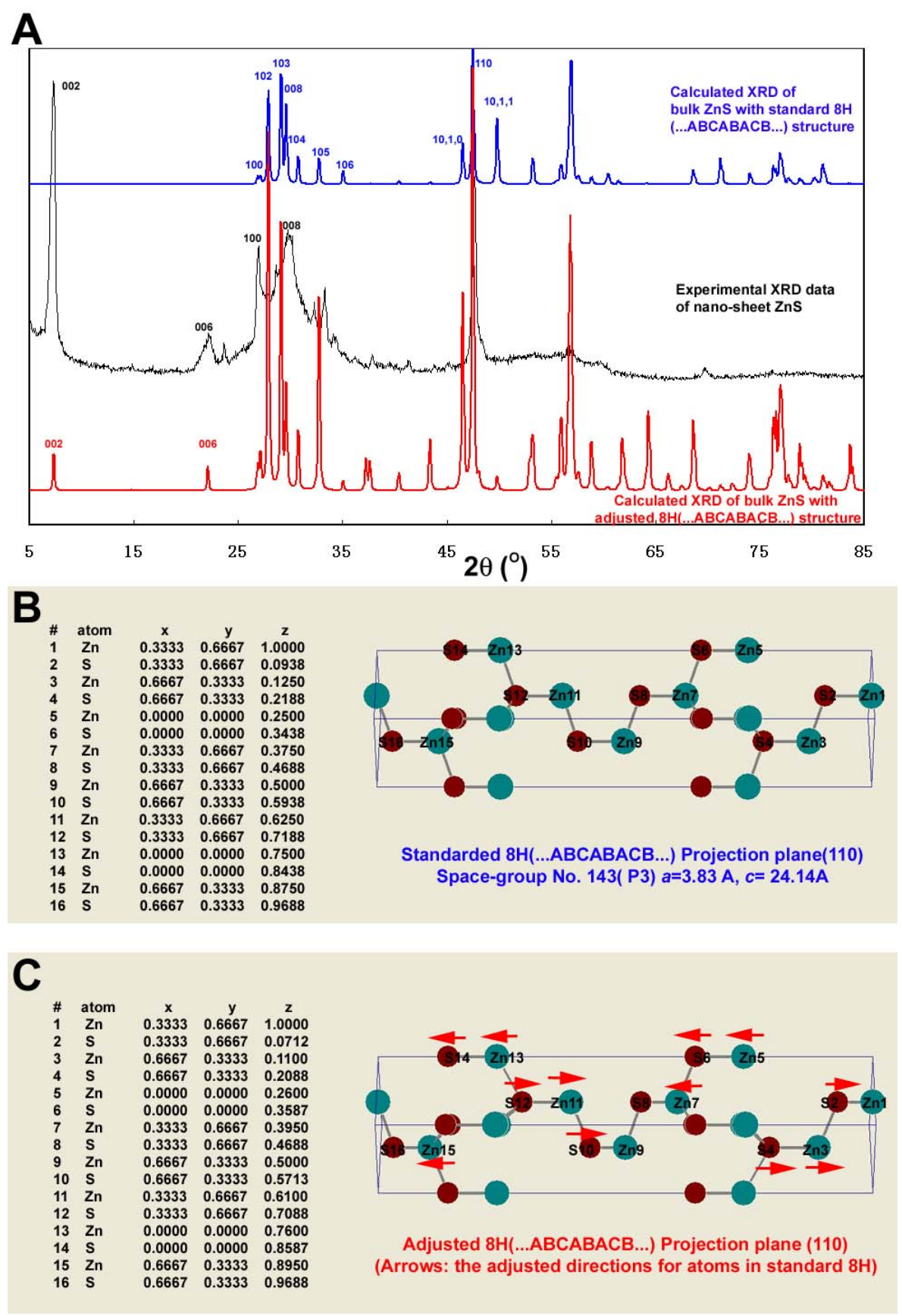

Supplementary Figure S2 a) Comparison of experimental x-ray diffraction (XRD) pattern from the ZnS nanosheets with the theoretical XRD patterns for bulk ZnS with one wurtzite $8 \mathrm{H}$ polytype structure (...ABCABACB...), and for bulk $\mathrm{ZnS}$ with a modified version of the same $8 \mathrm{H}$ polytype. b) \& c) The atomic positions and unit cell structure of the standard and modified $8 \mathrm{H}$ structures. The nanosheet shape and orientation with respect to the crystallographic axes is indicated below. 
The indexing of experimental peaks using the standard 8H polytype structure gives fair agreement, except for the presence in the experimental pattern of reflections that are extinct in the standard $8 \mathrm{H}$ structure.

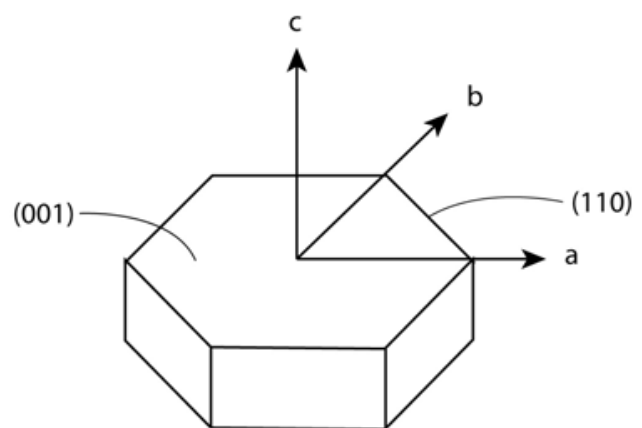

However, while the $8 \mathrm{H}(002)$ and (006) peaks are forbidden for the standard $8 \mathrm{H}$ structure, these peaks can appear as a result of small adjustments of the atom positions in $8 \mathrm{H}$, and we conclude that the $\mathrm{ZnS}$ nanosheet may possess a modified $8 \mathrm{H}$ polytype structure. In addition, the nanosheet $c$ distance is reduced to $24.14 \AA$ from the standard value of $25.04 \AA$.

Our structural analysis is incomplete, as the trial modified structure predicts the presence of additional reflections that are not seen in the experimental data: the (103), (104) and (105) peaks. Further analysis will be required to refine the atomic positions in the unit cell, and to incorporate the strong anisotropic shape and preferred orientation effects.

\section{Pair distribution function (PDF) analysis of the nanosheet structure}

The structure transformation in ZnS was further studied with synchrotron-based wide angle x-ray scattering (WAXS) measurements to high momentum transfer, from which real-space pair distribution functions (PDF), denoted $G(r)$, were obtained using standard methods [S1, S2]. As shown in Supplementary Figure S2, the PDF distinguishes ZnS cubic-hexagonal polytypism. The nanosheet PDF is most closely resembles a $8 \mathrm{H}$-wurtzite polytype structure in terms of peak position and intensity, rather than sphalerite or alternative wurtzite $2 \mathrm{H}$, $4 \mathrm{H}$ or other $8 \mathrm{H}$ polytypes. This result is show in Supplementary Figure S4. 


\section{Pair distribution functions of bulk ZnS standards.}

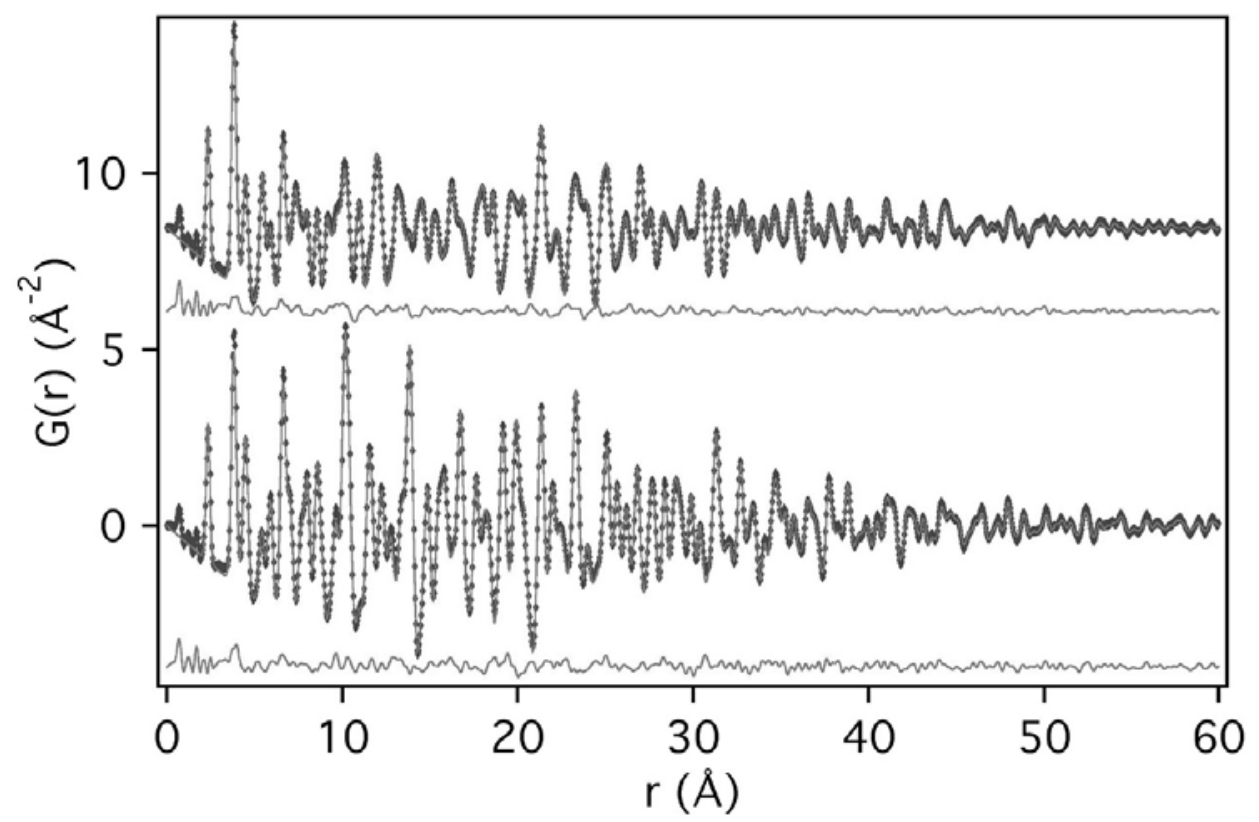

Supplementary Figure S3 Experimental (dots) and simulated (lines) pair distribution functions (PDF) for bulk ZnS with the standard $2 \mathrm{H}$ wurtzite (top) and the sphalerite (bottom) structures. As shown by the residuals beneath each curve, the PDF approach is highly quantitative and sensitive to ZnS cubic-hexagonal polytypism. The decline in PDF peak intensity with increasing interatomic distance, $r$, is an experimental effect of finite angular resolution at the $\mathrm{x}$-ray detector. This effect is constant for all samples.

\section{Experimental vs. simulated PDFs of the nanosheet structure}

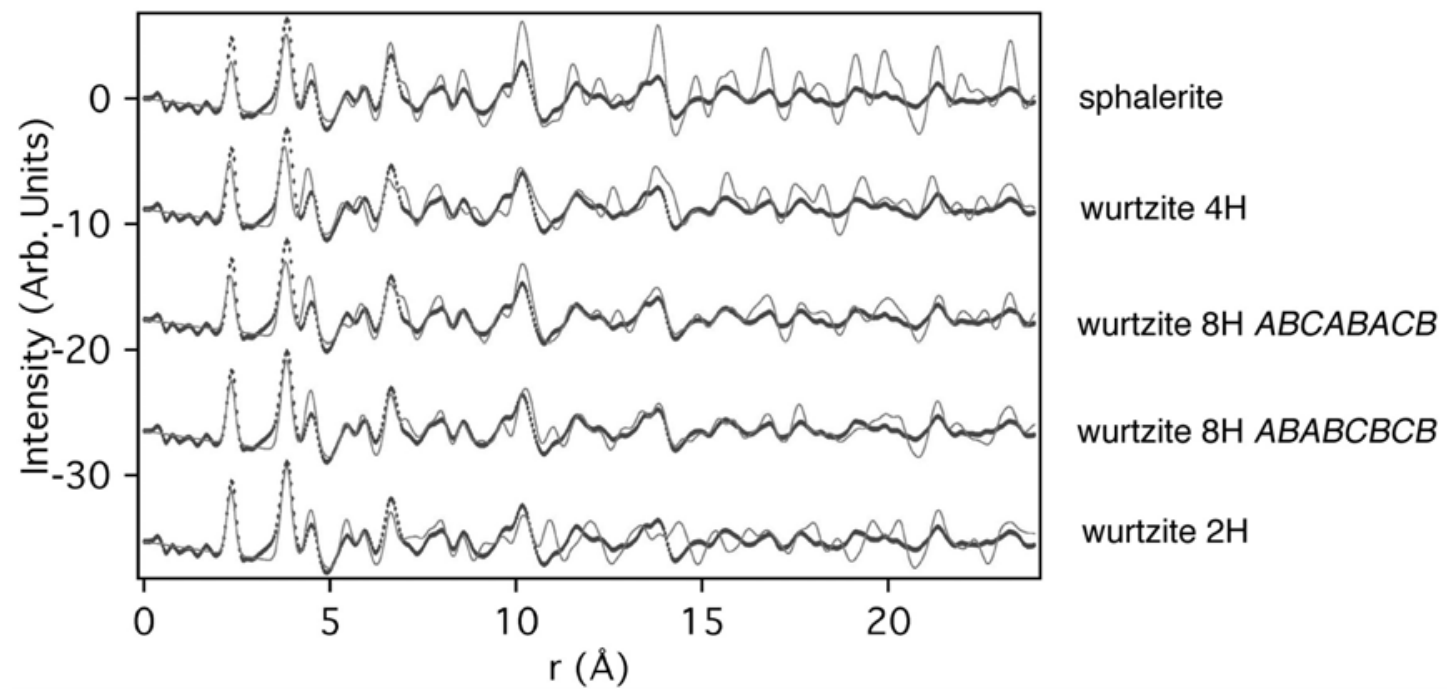

Supplementary Figure S4 Comparisons between the experimental PDF of nanosheet ZnS (dark markers) with simulated PDF patterns for a range of ZnS polytypes (light lines). The wurtzite $8 \mathrm{H}$ polytype with the $\{\ldots A B C A B A C B . .$.$\} stacking sequence gives$ the closest match to the experimental data in terms of PDF peak position and intensity. The agreement is not sufficient to conclude that the nanosheet structure contains this particular regular $8 \mathrm{H}$ stacking sequence, but indicates that the nanosheet has an associated polytype structure. 
[S1] B. J. Thijsse, J. Appl. Cryst. 17, 61 (1984)

[S2] S. J. L., Billinge, M. F.Thorpe (Eds.), Local Structure from Diffraction (Plenum, New York, 1998) .

X-ray scattering data were acquired at beamline 11-ID-C of the Advanced Photon Source (APS), and we thank Yang Ren. Use of the APS was supported by the U. S. Department of Energy, Office of Science, Office of Basic Energy Sciences, under Contract No. W-31-109-ENG-38.

\section{Temperature dependence of $\mathrm{ZnS}$ nanosheet thickness}

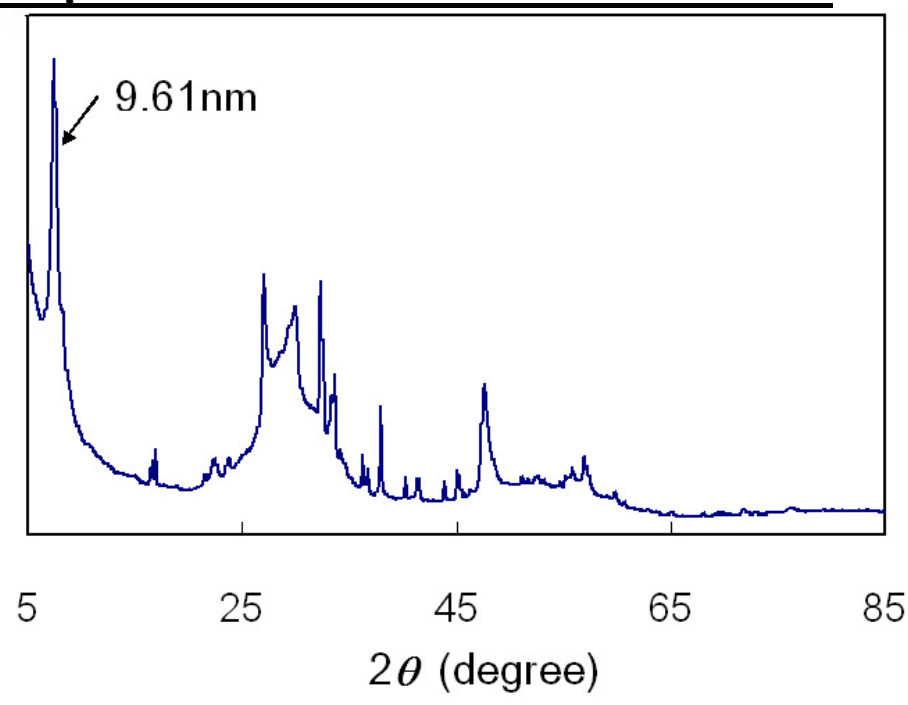

Supplementary Figure S5 X-ray diffraction pattern from ZnS nanosheets synthesized at $250{ }^{\circ} \mathrm{C}$. From the width of the (002) peak, the nanosheet thickness calculated with the Scherrer equation is $9.6 \mathrm{~nm}$.

\section{Thermodynamically stable ZnO nanoparticles}

Preliminary experiments show that $\mathrm{ZnO}$ oxide nanoparticles can be formed at the expense of bulk $\mathrm{ZnO}$ under hydrothermal conditions with high surface adsorption.

\section{$\underline{\text { TEM imaging and diffraction analysis of } \mathrm{ZnO} \text { nanoparticles }}$}



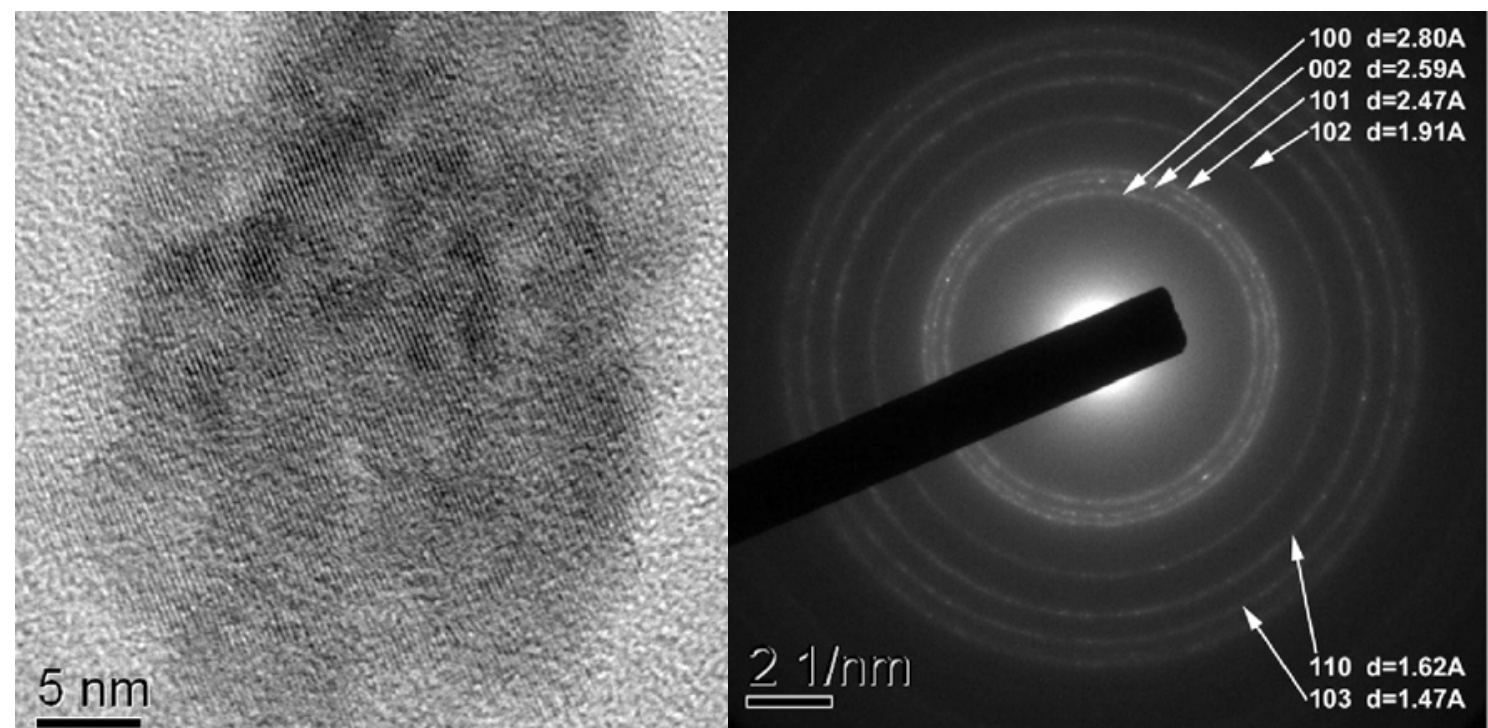

Supplementary Figure S6 Left. Transmission electron microscope image of an aggregate of $\mathrm{ZnO}$ nanoparticles formed during the hydrothermal treatment of bulk $\mathrm{ZnO}$. Right. Selected area electron diffraction pattern from $\mathrm{ZnO}$ nanoparticles. Diffraction rings are indexed in the wurtzite structure, and the broad line width confirms nanoscale particle dimensions.

\section{$\underline{\text { EDS analysis of } \mathrm{ZnO} \text { nanoparticles }}$}

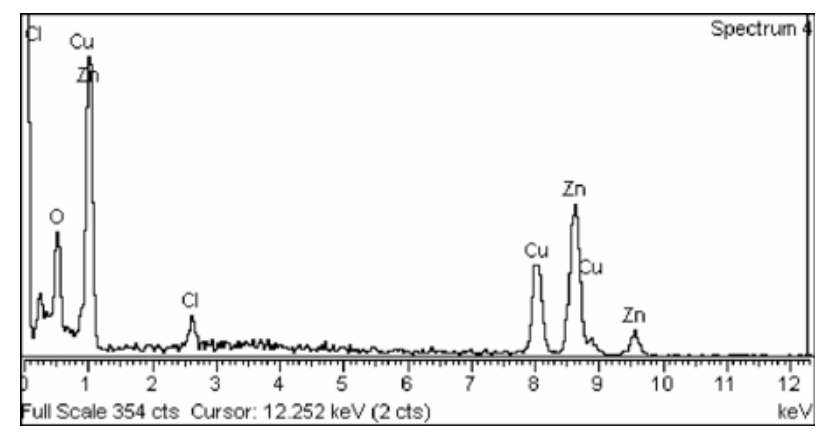

\begin{tabular}{l|ll}
\hline Element & Weight\% & Atomic\% \\
O K & 5.32 & 18.25 \\
Cl K & 2.12 & 3.28 \\
Cu K & 32.63 & 28.17 \\
Zn K & 59.93 & 50.30 \\
& & \\
Totals & 100.00 & \\
\hline
\end{tabular}

Supplementary Figure S7 Left. Energy dispersive spectroscopy (EDS) data from ZnO nanoparticles. Copper signal originates from TEM grid. Right. Table of elemental composition. 